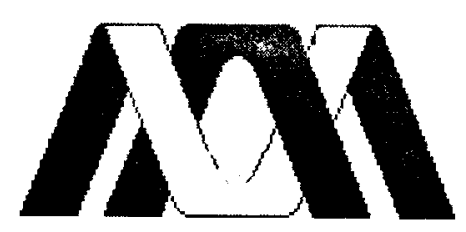

Casa abierta al tiempo

UNIVERSIDAD AUTÓNOMA METROPOLITANA

UNIDAD IZTAPALAPA

DIVISIÓN DE CIENCIAS SOCIALES Y HUMANIDADES

POSGRADO EN CIENCIAS ANTROPOLÓGICAS

Cultura, globalización y desastres en regiones vulnerables

Gabriela Vera Cortes

\title{
ENSAYO
}

PARA OBTENER EL DIPLOMA DE ESPECIALIZACIÓN

EN ANTROPOLOGÍA DE LA CULTURA

3

Director: Dr. Nestor García Canclini

México, D.F. 


\section{Cultura, globalización y desastres en regiones vulnerables}

Gabriela Vera Cortés

\section{Introducción}

El estudio de los mal llamados "desastres naturales" ha aumentado en los últimos años debido, en gran parte a los grandes desastres ocurridos en el mundo: El sismo de Guatemala, las sequías en el norte de Africa, el terremoto de Kobe, la erupción del Nevado de Ruíz, el Sismo de México y el huracán Mitch, recientemente. México se vio presionado por instituciones internacionales para buscar mejores medidas en la mitigación de desastres a partir del sismo de 1985. De tal forma que en 1986 se crea el Sistema Nacional de Protección Civil (SINAPROC) y en 1988 el Centro Nacional para la prevención de desastres (CENAPRED), órgano encargado de realizar investigaciones y monitoreo sobre desastres naturales).

Posteriormente otros desastres como el Huracán Gilberto en 1989, la explosión del Sector Reforma en Guadalajara y el Huracán Paulina en las Costas de Guerrero y Oaxaca, entre otros, han impulsado una mayor participación de investigadores y presencia de ONGs , así como de una mayor participación social en el tema. Sin embargo, uno de los problemas que no permite una respuesta eficaz para la mitigación de los desastres se encuentra en la visión teórica que tienen las autoridades sobre el mismo.

De eso tratará la primera parte de este ensayo, entender las diferentes perspectivas en el estudio y estrategias de acción contra los desastres puede permitir entender mejor el problema al que nos enfrentamos. Así mismo, se mostrará como la globalización acentúa una mayor polarización entre la población mundial, llevando a una mayor exclusión y por 
tanto a la agudización de la vulnerabilidad social, elemento fundamental en el perfil de los desastres.

En la segunda parte del ensayo se analizarán los cambios provocados por la globalización y como está agudizando y magnificando la vulnerabilidad social, de por sí siempre presente en las zonas marginadas. El estudio de la vulnerabilidad social en los desastres es un aspecto crucial en el estudio pues permite entender el perfil e incluso intensidad del desastre.

Finalmente, la tercera parte atenderá el papel de la cultura y percepción del riesgo entre los diferentes actores en el desastre. El nuevo papel que el Estado debe tener dentro de este nuevo orden mundial, así como el de las diferentes instancias civiles en la gestión del desastre.

\section{¿Que son los desastres?}

En el estudio de los desastres existen muchas conceptualizaciones al respecto y todo depende del interés del grupo de científicos o instituciones: Para Jesús Manuel Macías: "el desastre es una condición en la que parte de una sociedad sufre cambios producidos por uno o varios efectos destructivos ocasionados por fenómenos naturales o tecnoindustriales. Es un proceso condicionado por la vulnerabilidad social respecto a determinados riesgos". ${ }^{1}$

Ciertamente los desastres tecnoindustriales han aumentado en número a lo largo de todo este siglo y por otro lado se han presentado grandes desastres producidos por fenómenos naturales, tales como sequías, inundaciones, huracánes, etc los cuales han servido como detonantes. Estas dos definiciones forman parte de la "visión alternativa". Las causas más

\footnotetext{
1 Jesús Manuel Macías; Desastres y protección civil. Problemas sociales, políticos y organizacionales; Ciesas, México, 1999; p. 26.
} 
importantes y que le darán perfil al desastre se encuentra precisamente en las condiciones sociales, en su forma de organización, y por supuesto, forma parte de un proceso en el que la población se va tornando vulnerable y es menos apta para hacerle frente a un fenómeno natural o tecnoindustrial.

Así el desastre es multicausal, una de las causas, es el fenómeno natural que funciona como detonante. El resto de las causas se encuentran en el tipo de organización socioeconómica y en lo que éste grupo de científicos a denominado vulnerabilidad social: "Tiene raíces históricas colectivas y expresiones espaciales que dan lugar a la consideración del riesgo en diferentes escalas, pero con sobredeterminación social". ${ }^{2}$ El enfoque se centra en el análisis de la vulnerabilidad social y permite entender al desastre como un proceso.

Es importante mencionar que han surgido algunos teóricos del desastre en décadas pasadas, algunos de los cuales se han retomado en México. En la década de 1990 un grupo de investigadores de diferentes países latinoamericanos deciden formar La Red de Estudios Sociales en Prevención de Desastres en América Latina (la Red), una institución enfocada a estudiar a los desastres, sin embargo en los últimos años se encuentran en un periodo de letargo. Para latinoamérica esta organización ha sido importante pues ha permitido la publicación, desarrollo, traducción y divulgación de otro enfoque sobre el estudio del desastre.

Por otro lado, existe lo que se le ha denominado "visión dominante". Es dominante porque existe una mayor difusión, publicación, apoyo económico y es la visión que ha sido acogida por gran parte de los Estados en el mundo, ${ }^{3}$ es con esta visión que se le hace frente a los

\footnotetext{
2 Ibid. p. 26.

3 Kenneth Hewitt; The Idea of calamity in a Technocratic age. En k. Hewitt (Ed), Interpretations of Calamity; pp. 3-32, Mass.: Allen \&Unwin.
} 
desastres de una manera desafortunada. Además las instituciones creadas por parte de Estado como CENAPRED y Protección Civil en México, tienen esta visión.

La visión dominante de los desastres naturales es entendida como el resultado de procesos geofísicos extremos, dependientes de la naturaleza de las tormentas, terremotos, inundaciones y sequías. El tipo de investigación está enfocada en el monitoreo geofísico y pronósticos. El desastre es atribuido a la naturaleza y son considerados como eventos inesperados, meros accidentes. El sentido de la causalidad va del medio ambiente físico hacia sus impactos sociales. No se puede concebir que dentro del desarrollo de la modernidad en una sociedad se puedan observar acciones humanas orientadas a la destrucción y colapso de instituciones, por ello sólo se puede concebir como accidentes. La forma de solucionar es la intervención plena del gobierno y militarización de la zona. ${ }^{4}$ Modelo que surge desde la segunda guerra mundial y que se ha mantenido hasta la fecha, en diferentes países. ${ }^{5}$

Pero existe otro trasfondo: la visión dominante constituye una aproximación tecnocrática. La tecnocracia no sólo está obsesionada por la tecnología en el sentido estricto de estructuras de ingeniería y máquinas. Así la visión dominante adapta muy bien aquellas instituciones que realizan estudios técnicos, planes de desarrollo técnicos. ${ }^{6}$ El caso de México es fiel reflejo de lo que plantea Hewitt. El Centro Nacional para la Prevención de Desastres (CENAPRED), tiene esa perspectiva.

Para Hewitt, la calamidad natural es unos de los problemas especiales y es especialmente intratable para la tecnocracia. "Estamos atorados en muchas maneras en una forma de

\footnotetext{
4 Idem.

5 Russell R. Dynes; "La Planificación de emergencias en comunidades: falsos supuestos y analogías inapropiadas", en Cuadernos de Extensión; BUAP, CUPREDER; México; No. 2, Julio de 1999; pp- 7-9.

6 Idem
} 
estrategia tecnocrática que es particularmente arcaica e inflexible. La calamidad desafía directamente nuestras nociones del orden, ambas amenazan con ser interpretadas como un "castigo" por el desorden y la inutilidad de la ciencia", pueden ser vistas como claros límites al conocimiento y al poder, porque son iniciados de una manera en que son incontrolables para la sociedad. El mismo lenguaje es utilizado para mantener un sentido de discontinuidad, lo separa de la vida cotidiana y de la sociedad. Los desastres se vuelven así, fenómenos inmanejables, inciertos. No son vistos como espectro de las relaciones hombremedio. Se ha inventado su campo de problematización para adaptarlo a su conveniencia. Todo ello responde a la idea de modernización. Además de que mantener esta idea es conveniente, pues el Estado se lava las manos en la parte de responsabilidad que le corresponde al dejar que se profundice aun más la vulnerabilidad social.

La visión dominante no pueden contemplar las acciones humanas como orientadas a la destrucción, al colapso de las instituciones o a la desorganización de la economía en el espacio. Una actividad que directamente invita al desastre no podría ser intencionalmente puesta en el lugar, excepto por accidente y así se considera. No se puede aceptar una devastación dentro de un mundo racional, eso sólo lo puede hacer un criminal o un loco. ${ }^{7}$ "Estas interpretaciones tienden a ser uniformadoras, evolucionistas y normativas. Se articulan con una visión de la vida humana como especialmente progresiva. El desastre sugiere retrocesos más bien que progreso. La grave degradación del hábitat sugiere involución y aun la amenaza de la extinción". 8

\footnotetext{
${ }^{7}$ Idem.

8 Idem.
} 
La explicación anterior representa un panorama general sobre lo que existe en la investigación sobre desastres y permite entender cuales son las acciones tomadas por las autoridades.

El papel del Estado como órgano preocupado por ejercer el "control" y de intentar mantener el mismo orden social ha sido la constante en las acciones encabezadas durante la emergencia y reconstrucción. Intenta un control de la población al desplazar a los damnificados a los albergues.

Debe tenerse en cuenta que el desastre afecta a la población de una manera diferenciada, porque la vulnerabilidad social es diferenciada. Para entender mejor la idea, WilchesChaux, desagrega la vulnerabilidad social, para permitirnos entender mejor lo que abarca el concepto: Existe así una vulnerabilidad: social, económica, cultural, ideológica, técnica, ecológica, educativa, institucional y física. ${ }^{9}$

La vulnerabilidad social provoca que una población sea capaz o no de hacerle frente a un fenómeno natural o tecnoindustrial. Mientras más vulnerable se sea, el detonante del desastre puede ser una simple lluvia, como ha ocurrido ya en varias partes del mundo. La vulnerabilidad lleva también a que la recuperación del desastre sea mucho muy difícil.

\section{Lo global y lo local}

Los cambios ocurridos en las últimas décadas han provocado una mayor conexión de diferentes partes del mundo, de tal forma que lo global afecta a lo local en casi todos los ámbitos. Existen varios teóricos sobre la globalización, en este apartado se ha retomado a Manuel Castells, Giddens, Senneth y Ulrich Beck, todos ellos sociólogos, para mostrar

\footnotetext{
9 Wilches-Chaux; "La vulnerabilidad global"; en Los desastres no son naturales; La Red; Colombia; 1993; pp. 9-50.
} 
cuáles son los cambios más importantes. Además, parece ser que es entre los sociólogos, que se ha despertado un enorme interés por la denominada sociedad del riesgo. Manuel Castells, es sin embargo, el autor que hace un primer intento por sintetizar la mayor parte de los elementos que conforman la globalización.

La globalización está generando una mayor desigualdad, mayor exclusión y por tanto mayor vulnerabilidad social. Caldo de cultivo para los desastres tanto naturales, como técno industriales.

\section{Características de la Globalización}

Se ha escrito mucho acerca de los efectos de la globalización, me parece importante retomar algunos puntos que señala Manuel Castells, como conformadores del proceso de globalización.

Para Castells se está dando una nueva estructura social que se manifiesta de formas distintas, "depende de la diversidad e instituciones de todo el planeta. La nueva estructura social está asociada con el surgimiento de un nuevo modelo de desarrollo: el informacionalismo (atributo de una forma específica de organización social en la que la generación, procesamiento, transmisión de la información se convierten en fuertes fundamentales de productividad y del poder)". ${ }^{10}$

Los procesos de generación de conocimiento, la productividad económica, poder político/militar y medios de comunicación ya han sido profundamente transformados por el paradigma informacional. Están enlazados por redes globales de salud, poder y símbolo que funciona según ésta lógica. De ésta forma, las empresas dominantes, núcleos estratégicos

\footnotetext{
${ }^{10}$ Manuel Castells; La nueva era de la información. Economía, sociedad y cultura; siglo XXI; Vol 1, México; 1999; p. 40.
} 
de todas las economías se encuentran ya conectados al mercado mundial y su destino está en función de sus resultados en él. ${ }^{11}$

Existen, sin embargo, otros segmentos que no están conectados del todo, sectores económicos, sociedades locales desconectadas de estos procesos de acumulación y consumo que caracterizan a la economía/global. ${ }^{12}$

A nivel mundial existen nuevas condiciones de flexibilidad ilimitada que no atañe solo a la población cualificada. Existe una enorme movilidad en el trabajo, salarios y tiempo de vida laboral se reducen,

"El ascenso del capitalismo informacional global se caracteriza por el desarrollo y subdesarrollo económicos simultáneos y la exclusión e inclusión sociales...Existe polarización en la distribución de la riqueza a escala global, una evolución diferencial de la desigualdad de la renta en el interior de los países y un aumento sustancial de la pobreza y la miseria en el mundo en general y en la mayoría de los países, tanto desarrollados como en vías de desarrollo"13

Me parece importante destacar estos puntos, que el actor desarrolla a los largo de 3 volúmenes. He retomado sólo algunos, que me parece importantes para referirme a la exclusión de varios segmentos de la población Una exclusión que puede ser entendida también en términos de vulnerabilidad social, como se ha expuesto en la primera parte del ensayo.

Ciertamente la exclusión ha existido siempre, ha sido entendida de diferentes maneras, se han utilizado otros términos, como el de desigualdad social, marginación y también vulnerabilidad social. Pero los procesos de exclusión y de polarización se daban de una

\footnotetext{
11 Ibid. pp. 93-176.

12 Idem.
} 
manera diferente, el tipo de relaciones de poder era explicado a lo largo de un proceso histórico. La globalización impone nuevas reglas, reglas nunca claras. Los procesos de exclusión ocurren con una velocidad mucho mayor, una enorme flexibilidad en el trabajo y una incertidumbre creciente como parte de la nueva dinámica de la sociedad.

Manuel Castells define la exclusión social actual como "el proceso por el cual a ciertos individuos y grupos se les impide sistemáticamente el acceso a posiciones que les permitirán una subsistencia autónoma de los niveles sociales determinados por las instituciones y valores en un contexto dado".

La globalización está afectando de una manera diferenciada, no existe una claridad en las reglas del juego. La mayor flexibilidad laboral introduce a escena un nuevo concepto: la incertidumbre. Hasta el momento se han retomado algunos elementos que conforman la globalización, elementos que permiten entender la exclusión y que serán motivo de estudio en la investigación sobre la vulnerabilidad social.

En la globalización encontramos procesos diferenciados ciertamente, de tal forma que se puede hablar de los "espacios de flujo" y "espacios de lugar"14. Espacios que se han transformado, se perciben y se viven de una manera diferente. Ambos forman parte de una construcción social, sin embargo, la diferencia es que permanecer en el espacio de lugar tiene consecuencias negativas. Es formar parte de los segmentos excluidos de la población. La inclusión y exclusión son característicos de la globalización. En ambos encontraremos también ciertos elementos característicos: la incertidumbre, en los espacios de flujo los capitales se mueven a una velocidad vertiginosa, el mismo concepto tiempo cambia, tenemos la incertidumbre, perdida o ganancia de capital, incertidumbre en el trabajo,

\footnotetext{
13 Ibid; Vol III; p. 107.

14 Términos utilizado por Manuel Castells. Vol I. pp. 409-462.
} 
mayor migración, un desempeño laboral donde se empieza a trabajara mayor edad y se termina más joven. Reducción de sueldos. Las desventajas, sin embargo, son mayores para los grupos excluidos, tal parece que esa es la única regla clara. La polarización es más fuerte. El desempleo, sueldos muy bajos y que no alcanzan para reproducir la economía familiar, una familia que también está en un proceso de cambio, ya no es la tradicional familia nuclear, la absorción de un porcentaje mayor de mujeres con sueldos inferiores al de los hombres en términos generales, alcanzando en varios países de América latina de un $41 \%$ al $71 \%$ menos con relación al sueldo de los hombres. Las mujeres se vuelven más rentables, representan una mano de obra barata.

Hasta el momento hemos visto de manera general que está ocurriendo a nivel mundial y de esa oposición exclusión/inclusión. Como los procesos macro están influyendo en los micro, pero a la vez como éstos últimos le dan características específicas a lo local. Es preciso cambiar constantemente de escalas de estudio y entender como la globalización está afectando lo local.

Richard Sennett, utiliza otra escala de estudio, la del individuo. A partir de la experiencia de algunas personas dentro de los espacios de flujo, hace un análisis sobre las nuevas condiciones en el trabajo. En las urbes es más frecuente observar como un individuo cambia de casa y de trabajo varias veces en su vida, los conocimientos adquiridos a lo largo de toda una vida se vuelven obsoletos, como las acciones de larga duración que formaban parte de la generación anterior se transforma en la actualidad y como la incertidumbre llega a formar parte de la vida diaria. Y como estas acciones de corta duración llegan a afectar la 
vida persona, donde las relaciones sociales ya no son tan duraderas, gran parte de nuestra vida se vuelven acciones de corta duración y como esto afecta a nuestro carácter. ${ }^{15}$

El ejercicio de Sennett resulta muy interesante si lo aplicamos al estudio de los desastres. Tendíamos que reconceptualizar al desastre mismo. Las nuevas condiciones llevan a pensar que la población vive actualmente dentro de una emergencia ${ }^{16}$ y el resto al borde del desastre. Dadas las nuevas condiciones, inseguridad en el empleo, bajos salarios, una edad madura y una experiencia obsoleta, los individuos ingresan y egresan de la incertidumbre al desastre y viceversa. Hablamos de un desastre personalizado y frecuente. En las grandes urbes que forman parte de los espacios de flujo tendríamos que pensar en un desastre individualizado, cambiante y constante. A no ser que formen parte de los cinturones de miseria y por tanto de amplios sectores de población. Pero que ocurre en los espacios de lugar. En regiones excluidas, como por ejemplo, casi todo el continente africano. Donde primero se da el colonialismo, después el neocolonialismo y actualmente la exclusión como parte de esta globalización, lo anterior lleva a una frecuencia mayor de hambrunas, sequías, muertes, etc; donde el desastre parece que llegó para quedarse. Hablamos entonces de nuevos procesos de vulnerabilidad social que simplemente se acumulan.

En las regiones excluidas tendríamos que agregar a las condiciones de inseguridad en las que siempre ha vivido la población, las nuevas condiciones, un desgaste paulatino, pero constante a lo largo de siglos, aunado a proceso mas cortos, como el desempleo, subempleo, migración del campo a la ciudad, formación de cinturones de miseria, aumento del comercio informal, reducción de salarios, aumento en los precios de la canasta

\footnotetext{
${ }^{15}$ Richard Sennett; La corrosión del carácter. Las consecuencias personales del trabajo en el nuevo capitalismo; Anagrama, España, 2000. pp. 13-31.

${ }^{16}$ Para su estudio, el desastres ha sido divido en tres etapas: prevención, emergencia y reconstrucción. La emergencia representa el momento mismo del desastre.
} 
básica, abandono a la agricultura de subsistencia, reducción de subsidios, mayor flexibilidad laboral, debilitamiento de los sindicatos, disminución del papel del Estado en las decisiones económicas, una política social de tipo asistencialista, aumento de la pobreza y extrema pobreza.

La vulnerabilidad social ha aumentado desfavorablemente en las últimas décadas y la población vive en una incertidumbre que se ha vuelto cotidiana.

La exclusión ha aumentado los índices de marginalidad, la vulnerabilidad social es hoy cada día mayor, sin embargo, el estudioso de los desastres tiene que estudiar los dos tipos de vulnerabilidad que se están dando.

Aunque la globalización lleva a una dirección, la cultura, la política estatal, las condiciones históricas, a pesar de todo siguen dando una distinción particular a lo local y aunque en términos generales el camino sea una mayor pobreza y exclusión, los rostros de la miseria también tiene diferentes matices. En las sociedades más vulnerables, cualquier detonante puede provocar un desastre de consecuencias mayúsculas, la población no puede hacerle frente, viven en condiciones deplorables. La capacidad para recuperarse del desastre también es mínima. En la Ciudad de México todavía encontramos las consecuencias del sismo de 1985. Los países centroamericanos que sufrieron los embates del huracán Mitch tardarán muchos años en recuperarse, de hecho, muchos de los recursos de los Estados fueron desviados para atender las nuevas prioridades.

\section{Sociedades de riesgo}

Varios sociólogos han desarrollado en los últimos años el concepto de Sociedad del riesgo a propósito de la globalización y de los desarrollos tecnológicos. Comentan que el riesgo está presente en todo momento, cualquier decisión puede ser catastrófica, que las decisiones individuales pueden tener consecuencias graves para un grupo o para el mundo entero. 
Ellos se refieren al desarrollo en las zonas industrializadas y a los adelantos en la tecnología. Es el caso de Giddens y de Ulrich Beck. ${ }^{17}$

Sin embargo, no toman en cuenta el tipo de desarrollo de amplios sectores excluidos y que el tipo de organización socioeconómica va llevando a una pobreza desbordada. Ciertamente, también estos espacios son afectados por las decisiones que se tomen en estos espacios de flujo, industrializados y tecnificados. Pero también se dan procesos propios, locales, que aunque influidos por lo global, las características locales terminarán caracterizando la región.

El concepto de riesgo es diferente entonces. Y sin embargo la globalización ha ayudado a crear un mayor número de pobres. La miseria se vuelve el principal elemento para que podamos referirnos al riesgo.

\section{Cultura y percepción del riesgo}

Autores como Sennet nos dicen que el riesgo es manejado y sentido de una manera individual, se enlaza con la experiencia personal del trabajo, donde la experiencia ya no es útil, lo mismo que los conocimientos aprendidos durante años. "Es desprenderse del pasado y vivir en el desorden, son también maneras de vivir al límite" ${ }^{18}$ Lo anterior ocurre en ambos espacios, el desorden y el vivir en el límite se ha vuelto parte de la vida cotidiana, solo que la forma de enfrentarlo es diferente. En las regiones excluidas la experiencia persiste, la agricultura de subisistencia es una forma de vida, el haber vivido en la marginación se volvió en un aprendizaje para los grupos étnicos, aunque últimamente

17 Giddens, Anthony; La tercera Vía. La renovación de la socialdemocracia; Taurus, España; 1999; pp. 6894. Ulrich Beck; "Teoría de la sociedad del riesgo"; en Las consecuencias perversas de la modernidad; (Giddens, Baumann, Luhmann, Beck); Anthropos; España; 1996; pp. 201-222.

18 Ibid. p. 83. 
tengan que echar mano de una migración temporal o definitiva. La cultura misma provoca que se busquen diferentes estrategias, la vida se reinterpreta y se reinventa diariamente al vivir al limite.

El abandono de los campesinos por parte del Estado, la modificación del artículo 27, falta de créditos, suelos erosionados y en zonas accidentadas, falta de subsidios, ha provocado una migración forzosa para poder reproducir la economía familiar. Las remesas se han convertido en uno de los ingresos más importantes para nuestro país. Los indígenas tienen que enfrentar otros problemas, el lenguaje y la discriminación racial, la sobreexplotación forma parte de su vida cotidiana.

Estos grupos tienen que reinventarse cuando salen de estos espacios, pero cuando permanecen en ellos, la experiencia y cultura forma parte de su vida.

Hablamos entonces de diferentes espacios y también de diferentes tiempos. Ellos viven en diferentes tiempos, la sobrevivencia les exige transitar de uno en otro de manera frecuente. Dentro de su espacio hacen uso de su experiencia, fuera de el se reinventan.

México es un verdadero mosaico cultural, es decir, tienen una forma de percibir, entender y actuar en este mundo.

La percepción del riesgo es una construcción social que se relaciona estrechamente con la cultura misma, por lo mismo existirán tantas percepciones del riesgo como culturas existan. Douglas y Wildavsky consideran "la percepción de riesgo, primeramente como un fenómeno socio-cultural afectado por la organización y los valores sociales opuestos a algo definido según se afirma, como evaluaciones objetivas versus subjetivas." ${ }^{19}$

\footnotetext{
${ }^{19}$ Douglas y Wildavsky, Risk and Curlture, (1982); citado en Oliver-Smith, Perspectivas antropológicas en la investigación de desastres. p. 68.
} 
Los mismos autores señalan que ciertas amenazas son ignoradas mientras que otras llaman más la atención y son recibidas con mucha ansiedad. Así, establecen que grupos diferentes sostienen perspectivas diferentes sobre lo que se piensa es peligroso. ${ }^{20}$

Oliver Smith menciona que "la percepción y la evaluación del riesgo se basan en las normas y valores culturales que se dan dentro de las relaciones que las comunidades humanas tienen con sus entornos físicos y sociales". ${ }^{21}$

En muchas comunidades pequeñas ocurren desastres año con año. Desastres que no llaman la atención de la Televisión, así que quedan en el anonimato. Cada año, la zona del Pánuco se inunda y la población ha implementado algunas estrategias de sobrevivencia. La experiencia les ha ayudado ha hacerle frente a las inundaciones. Existe un amplio conocimiento de su entorno, producto de siglos de convivencia con la naturaleza. Su percepción del riesgo es otra. Para ellos la inundación no representa nada extraordinario, por tanto no es un desastre. El verdadero desastre está en perder el trabajo y las cosechas. La percepción del riesgo es un elemento que se construye socialmente y se transforma de acuerdo a la experiencia y cultura.

Ciertamente el papel de las autoridades es básico en el logro de la mitigación del desastre. Es con su atención, apoyo y diálogo que se puede tener un logro sustancial para salvar, en la medida de lo posible, el mayor número de habitantes. Pero no es con el autoritarismo, ni con dictar soluciones desde el escritorio, ni con intentar dar soluciones con propuestas estandarizadas para cualquier tipo de desastres. El Estado mismo se debe transformar.

Es necesario tener un conocimiento mucho mayor sobre la región para así aplicar medidas que realmente ayuden a la mitigación del desastre. La percepción del desastre, el entorno

\footnotetext{
20 Idem.

21 Ibid. p. 69.
} 
cultural y las características económicas de las comunidades son elementos que son necesarios tomar en cuenta en la toma de decisiones para la propia mitigación. La experiencia y el conocimiento de su entorno se vuelve trascendental.

Debemos recordar que los habitantes son actores no objetos pasivos. Además, son precisamente ellos quienes viven en riesgo, por lo tanto, tienen derecho a opinar, derecho que les ha sido negado. Es ahí, donde podrían trabajar las autoridades competentes, dentro de una coordinación entre los diferentes sectores, los cuales puedan opinar y ser escuchados y así, en conjunto se pueda encontrar la o las soluciones más viables.

De no hacerlo, se puede dar un caos en el momento de la emergencia. Y es muy probable, que parte de los habitantes tomen ciertas decisiones que muy probablemente no serán las que haya dictado "protección civil" durante la llamada emergencia.

En ésta parte, queremos hacer mención sobre la importancia de que las organizaciones artificiales, es decir, los comités de protección civil, deban ser legitimados por el pueblo para que sean aceptados.

...la lógica de organización es diferente si es inducida que si se sustenta en una historia comunitaria de participación. La tendencia pareciera ser a que las organizaciones inducidas desde las autoridades gubernamentales adquieren una lógica claramente utilitarista, muchas veces evitando sistemáticamente generar expectativas a nivel de los beneficios intangibles. Esto por lo general, permite que la participación alcance objetivos materiales de manera más o menos rápida, sin embargo alcanzando niveles de participación muy bajos y una tendencia acentuada hacia el estancamiento de la organización ${ }^{22}$.

\footnotetext{
${ }^{22}$ Arellano, David; Liliana Rivera; Tendencias innovadoras en la gestión de la participación social; Documentos de Trabajo, No. 67; CIDE; México, 1998; p. 24.
} 
No nos debe extrañar la participación social, en los últimos años se ha intentado ya, por ejemplo en los programas de Solidaridad y ahora con Progresa, donde para el logro de los objetivos propuestos se pide la participación organizada de la población y se han obtenido buenos resultados, aunque también muchas críticas:

La participación organizada de la sociedad ha aparecido en espacios locales de gobierno como un instrumento sustantivo para el desarrollo social. Se han diseñado, impulsado e implementado diversos programas de desarrollo social que parten de la premisa de que la participación organizada es sustantiva para el verdadero éxito de éstos problemas sociales, escapando de las lógicas estrictamente asistenciales ${ }^{23}$.

Lo anterior demuestra un nuevo camino que las autoridades están implementando. Así, que la propuesta que se sugiere, al buscar una mayor participación social para la mitigación del desastre y aún en todas las etapas del desastre, no es una propuesta realmente novedosa.

Creemos que la participación social en las diferentes fases del desastre es esencial para el logro de mejores resultados. La población de ninguna manera es un ente pasivo y en espera de que le solucionen todos sus problemas.

Por otro lado, es importante demostrar como el tema de la mitigación del desastre se relaciona estrechamente con la vida cotidiana, pues las mismas autoridades, sin proponérselo están ocasionando la agudización de conflictos entre los partidos políticos, lo cual lleva a una división de la comunidad.

\footnotetext{
${ }^{23}$ Ibid. P. 9.
} 
Lo anterior debe suponer, por parte de las autoridades estatales y federales, una búsqueda en el fortalecimiento de la organizaciones comunitarias de base, entendiendo que las forma de participación pueden jugar un papel sustancial en el logro de los objetivos.

Debemos buscar una mayor participación social; y entender por participación social a aquellos principios de solidaridad colectiva, donde se consideran los siguientes parámetros: una iniciativa surgida de la comunidad, que se sustente en organizaciones comunitarias, con intereses colectivos, políticos y económicos de corto y largo plazo presentes ${ }^{24}$ en sus estrategias de acción, y que genere, además una colaboración gobierno-sociedad en trabajo conjunto .

Existe una confrontación y estrategias de lucha en los diferentes espacios. Si en el espacio de flujos el individuo es el que se enfrenta a la incertidumbre, en el otro espacio es un grupo. En algunos lugares la estrategia ha sido transformar la estructura familiar, de ser una familia nuclear hoy es extendida.

Si el Estado sigue manteniendo esa "visión dominante" explicada en la primera parte de este texto encontrará "supuestas" soluciones que no darán una solución real al problema de la vulnerabilidad social. El problema no está en el fenómeno natural sino en la organización misma. El mismo Estado, como institución, representa parte de la vulnerabilidad y parte del problema. El Estado también está buscando nuevas vías de acción, nuevas formas de enfrentar los cambios que lo han debilitado, pero aún así su papel es trascendental.

24 Ibid. P. 12-13. 